CAHIERS DE

NARRATOLOGIE

\section{Cahiers de Narratologie}

Analyse et théorie narratives

$21 \mid 2011$

Rencontres de narrativités : perspectives sur l'intrigue musicale

\title{
La Narrativisation de la musique
}

La musique : récit ou proto-récit?

Jean-Jacques Nattiez

\section{(2) OpenEdition}

Journals

Édition électronique

URL : http://journals.openedition.org/narratologie/6467

DOI : $10.4000 /$ narratologie. 6467

ISSN : 1765-307X

Éditeur

LIRCES

Référence électronique

Jean-Jacques Nattiez, «La Narrativisation de la musique », Cahiers de Narratologie [En ligne], 21 | 2011, mis en ligne le 21 décembre 2011, consulté le 19 avril 2019. URL : http://journals.openedition.org/ narratologie/6467 ; DOI : 10.4000/narratologie.6467

Ce document a été généré automatiquement le 19 avril 2019

Article L.111-1 du Code de la propriété intellectuelle. 


\title{
La Narrativisation de la musique
}

\author{
La musique : récit ou proto-récit?
}

Jean-Jacques Nattiez

\section{La musique raconte-t-elle une histoire?}

1 Lorsque j'étais enfant et que l'on me demandait de jouer un morceau au piano pour les visiteurs de mes parents, il n'était pas rare qu'on ajoute : «Mais tu nous diras d'abord ce que ça raconte !», prenant pour acquis que toute musique est narration d'une histoire ${ }^{1}$.

2 Sans doute parce que la musique, à la différence de la peinture et de la sculpture, partage avec le récit et le film une dimension linéaire; parce que la présence de titres, y compris de titres narratifs, dans le répertoire occidental, incite l'auditeur à entendre dans toute musique un récit; parce que les personnes qui n'ont pas eu d'éducation musicale se sentent plus à l'aise si une œuvre musicale est rattachée à la réalité du monde vécu et qu'ainsi, elle leur "parle». Une série d'expériences menées par François Delalande à propos de La Terrasse des audiences du clair de lune de Debussy et qui visait à identifier quelles étaient les conduites d'écoute des auditeurs, avait fait la démonstration que la conduite narrative était une de celles qui avait été spontanément adoptée par les sujets (Delalande 1989: 79). La raison est simple, et l'ouvrage fondamental de Jean Molino et Raphaël Lafhail-Molino sur le récit littéraire le montre à l'évidence : l'être humain n'est pas seulement, dans son essence anthropologique, un Homo symbolicus ou un Homo ludens, mais aussi un Homo fabulator, toujours prêt à intégrer dans un récit les objets ou les actions qui s'offrent à nos sens selon une succession linéaire : « La fonction fabulatrice est une faculté fondamentale de l'humanité » (Molino, Lafhail-Molino 2003 : 315). Lorsque nous mettons en branle une conduite narrative pour établir un lien entre les objets d'une chaîne linéaire, notamment non-linguistiques, c'est une intrigue que nous construisons, et j'en rappellerai l'excellente définition fournie par Paul Veyne pour caractériser l'écriture de l'histoire :

Les faits n'existent pas isolément, en ce sens que le tissu de l'histoire est ce que nous appellerons une intrigue, un mélange très humain et très peu « scientifique " de causes matérielles, de fins et de hasards; une tranche de vie, en un mot, que l'historien découpe à son gré et où les faits ont leurs liaisons objectives et leur 
importance relative : la genèse de la société féodale, la politique méditerranéenne de Philippe II ou un épisode seulement de cette politique, la révolution galiléenne. Le mot d'intrigue a l'avantage de rappeler que ce qu'étudie l'historien est aussi humain qu'un drame ou un roman, Guerre et Paix ou Antoine et Cléopâtre. (Veyne 1971:46)

3 L'attitude de nombreuses personnes, amateurs de musique ou non, justifie sûrement que j'examine, même rapidement, en quoi il est légitime ou non de parler de " récit musical ». De plus, le développement, dans la musicologie des vingt dernières années, de recherches dites «narratologiques" dont je traiterai à la fin de cet article, en est une raison supplémentaire ${ }^{2}$. Mais il y a plus aujourd'hui : la psychologie cognitive et la neurobiologie de la musique se sont plus récemment penchées sur la capacité des auditeurs de musique à narrativiser la musique. Je renvoie tout particulièrement aux deux numéros spéciaux de la revue Musicae Scientiae: "Rhythm, Musical Narrative, and Origins of Human Communication " (Trevarthen, 1999-2000) et "Narrative in Music and Interaction" (Imberty-Gratier, 2008) auxquels j'emprunterai tout à l'heure des éléments. Mais pour examiner les possibilités de narrativisation de la musique, il convient tout d'abord de tenter de définir ce qu'est un récit littéraire.

Nous sommes aidés en cela par l'ouvrage de Jean Molino et Raphaël Lafhail-Molino cité à l'instant. Pour les auteurs, un récit est d'abord caractérisé par « une phrase d'action, centrée autour d'un verbe exprimant une situation dynamique (acte ou action): "La marquise sortit à cinq heures", "Julien Sorel posa sa main sur celle de Madame de Rênal", "Charles Bovary entra dans la classe" » (Molino, Lafhail-Molino 2003 : 24). En musique, faites se succéder deux événements sonores contrastés, et, placés ou se plaçant dans une situation d'écoute narrative, les auditeurs tenteront d'établir un lien entre les deux en construisant une intrigue pour les relier. Mais, en règle générale, il y a dans un récit littéraire davantage que cette "plus petite unité narrative», car un récit est un objet hiérarchiquement stratifié, organisé en séquences de phrases et en épisodes (ibid. : 33). Une deuxième analogie apparaît alors avec la musique. Une œuvre musicale est, elle aussi, faite d'un certain nombre d'événements musicaux, objets de récurrences et de variations, mais elle est également organisée hiérarchiquement en unités de différents niveaux: motifs, phrases, thèmes, etc.

5 Mais les auteurs ne s'en tiennent pas à cette caractérisation taxinomique et hiérarchique du récit. "Au cours du récit, actions et événements se succèdent de telle sorte qu'à chaque instant l'auditeur ou le lecteur se demande : qu'arrive-t-il ensuite? [...] Nous ne sommes enfin satisfaits que si le récit aboutit à une conclusion qui correspond à nos attentes et donne un sentiment de clôture. L'auditeur et le lecteur sont ainsi animés par la curiosité, surpris par des événements inattendus, pris par la tension d'un suspens et enfin apaisés par le dénouement. » (ibid. : 42-43) Ici, et je fais appel bien entendu à la tripartition sémiologique de Jean Molino (2009 : passim), on est passé de la caractérisation immanente des structures hiérarchisées aux conduites esthésiques des auditeurs et des lecteurs qui prennent en charge, tant dans le récit littéraire que dans les productions musicales, les unités découpées par la taxinomie et les phénomènes d'implication linéaires.

6 On aura immédiatement remarqué, en effet, que cette phrase portant sur le récit littéraire pourrait aussi bien s'appliquer à la perception de la musique en en changeant quelques mots, et, dans l'adaptation qui suit, je vais employer à dessein le vocabulaire mis 
de l'avant par Leonard Meyer, notamment dans Émotion et signification en musique (Meyer, 1956) :

Au cours du déroulement musical, des événements sonores se succèdent de telle sorte qu'à chaque instant l'auditeur peut se demander: et que vais-je entendre maintenant? Nous ne sommes satisfaits que lorsque les attentes créées par les répétitions, les suspensions, les tensions musicales trouvent enfin leur conclusion dans des points de repos, provisoires ou définitifs, et procurent un sentiment de clôture.

7 C'est donc une analogie esthésique qui aura pu inciter à la fois les amateurs de musique et des secteurs contemporains de la musicologie à parler de narration et de narrativité en musique.

\section{La narrativisation de la musique : la musique comme proto-récit}

8 Privée de tout accompagnement verbal - dans le titre, les annotations, un récit programmatique, une sollicitation expérimentale -, une œuvre musicale, par elle-même, ne peut pas nous raconter une histoire. Jamais une œuvre musicale ne nous dira quelque chose comme «Longtemps je me suis couché de bonne heure ». Sinon, il n'y aurait pas de différence entre la musique et le langage comme formes symboliques. Les compositeurs d'œuvres qu'ils veulent explicitement narratives le savent bien, qui font précéder leurs œuvres de programmes et de récits. Pensons à Berlioz qui demande expressément, en tête de la partition de la Symphonie fantastique, à distribuer son texte liminaire aux spectateurs, et à Dukas qui reproduit la ballade de Goethe en tête de L'apprenti sorcier, en souhaitant probablement qu'il soit publié dans le programme du concert. À n'en pas douter, dans les poèmes symphoniques, le titre et/ou le programme narratif font partie intégrante de l'œuvre. Privée du soutien linguistique, l'œuvre musicale n'est pas un récit, mais elle peut être un proto-récit, ce qui est tout différent. C'est ce que Mendelssohn avait bien compris en composant ses Romances sans paroles, et Adorno, en qualifiant telle symphonie de Mahler de « récit qui ne raconte rien » (1976:117).

9 Le « discours » musical s'inscrit dans le temps. Il est fait de répétitions, de rappels, de préparations, d'attentes, de résolutions. Si l'on est tenté de parler de récit musical, c'est à cause, non de son contenu intrinsèque et immanent, mais en raison des effets de l'organisation syntaxique de la musique, de la conduite narrative que commande la musique grâce aux jeux d'implications et de réalisations que Meyer a si bien décrits, notamment dans Explaining Music (1973, passim). Au niveau immanent et du point de vue narratif, ce que la musique peut faire de mieux, c'est d'imiter l'allure du langage et du récit. Par là-même, elle prend le statut d'un proto-récit.

Cela fait longtemps que les linguistes se sont penchés sur ce qu'ils ont appelé les " éléments musicaux » du langage humain. Musique et langage ont en commun d'être constitués d'objets sonores. Cela est particulièrement évident dans les langues dites à ton. Dans la musique de tambours d'une danse d'initiation au mariage, la danse Mbaga des Baganda de l'Ouganda (Nattiez-Nannyonga Tamusuza 2005 : 1119), l'un des huit motifs à la base de cette danse imite le contour intonatif et rythmique du mot «baakisimba » qui signifie: "Ils l'ont planté [le bananier]». On peut légitimement penser que, dans les sociétés où l'on utilise le tambour pour communiquer des messages linguistiques, l'insertion de ces motifs dans un contexte d'exécution musicale et chorégraphique 
s'accompagne, pour les auditeurs, du contenu sémantique que le même motif véhicule lorsqu'il est utilisé comme moyen de communication. Mais il faudrait, bien sûr, le vérifier par une enquête sur le terrain.

11 Dans le langage comme en musique, il y a des rythmes et des accents, des longueurs de notes et de syllabes, et ce n'est pas un hasard si, en grec, mousikê désignait la poésie lyrique, c'est-à-dire quelque chose qui était à la fois ce que nous appelons aujourd'hui musique et poésie, et il n'est pas impossible d'expliquer la dimension rythmique et métrique de la poésie lyrique moderne par ses liens indissociables, dans ses origines, avec la musique, comme l'ont souligné Molino et Tamine dans leurs propositions pour l'analyse de la poésie (1987). Mais il y a plus. Dans le langage, à l'exception des langues à ton, les voyelles n'ont pas de hauteurs fixes, mais l'enchaînement des syllabes crée des courbes intonatives - les prosodèmes - auxquelles certains phonéticiens - je pense en particulier à Pierre Delattre $(1966,1967,1969)$ - ont consacré des études spécialisées. Ceci est décisif : musique et langage partagent la linéarité du discours et l'utilisation d'objets sonores. La musique est capable d'imiter la courbe intonative d'un récit.

Dans le Quinzième quatuor de Beethoven, il n'est pas nécessaire d'avoir lu le motto publié en tête du dernier mouvement - » Muss es sein? Es muss sein » (Le faut-il ? Il le faut) - pour reconnaître, dès le début, que nous sommes en présence d'une question, suggérée par la quarte ascendante, suivie d'une réponse double, évoquée par les quartes descendantes. Et à partir de là, le reste du mouvement peut être interprété comme la transposition musicale d'un dialogue. Nous ne savons pas ce qui est dit, mais Beethoven nous dépeint le caractère de l'échange, un peu comme si nous en captions les inflexions à travers un mur ou que nous écoutions une conversation dans une langue que nous ne connaissons pas.

Les voix du quatuor ont donc bien ici le caractère de ce que Edward Cone, à propos du dialogue du hautbois et du cor anglais dans la "Scène aux champs» de la Symphonie fantastique de Berlioz, appelle des «personnages virtuels » ou, plus précisément dans sa terminologie, des « agents virtuels » (Cone 1974 : 88). C'est évidemment le mot « virtuel» qui est ici le plus important. La mélodie du basson, dans le Pierre et le loup de Prokofiev, peut devenir le personnage du grand-père, mais seulement une fois éclairé par un texte verbal qui lui assigne un sens dénotatif. Sans doute est-ce en raison de cette même dimension virtuelle que le vocabulaire traditionnel de l'analyse de la fugue utilise des termes comme "sujet ", "réponse ", " exposition », " discussion » et "sommaire », et que, à l'époque baroque, les théoriciens ont été nombreux, je pense notamment à Mattheson (1739), à tenter de retrouver dans la musique de son époque les différents moments du discours rhétorique. Il fait ainsi correspondre à l'exordium l'introduction, pas toujours présente ; à la narration et la divisio, l'exposition ; à la confirmatio et la confutatio, le développement; à la peroratio, la récapitulation et la coda. Dans un travail récent, Mihaela Corduban réexamine le premier livre du Clavier bien tempéré à partir de la terminologie rhétorique utilisée à l'époque (Corduban 2011).

14 Ces cas d'imitation de l'intonation ne sont pas uniques. Nettl (1958) a suggéré que l'accentuation de la première syllabe en tchèque explique le schéma accentuel de la phrase musicale des compositeurs tchèques. Robert Hall (1956) a cherché à retrouver l'influence des intonations de l'anglais dans la musique d'Elgar. Il écrit

15 En anglais britannique comme en anglais américain, la fin d'une phrase déclarative est caractérisée par une intonation descendante, d'un ton relativement haut à un ton relativement bas. C'est également ce qui se produit dans une question commençant par un interrogatif (par ex., Where are you going ?). Mais dans les questions qui ne commencent pas par un interrogatif (par 
ex., Are you coming ?), l'anglais américain et la plupart des langues européennes utilisent une intonation ascendante marquée, alors que l'anglais britannique a recours à la même intonation descendante que dans Where are you going?

Se tournant vers la musique d'Elgar, Hall rapporte que, chez lui - ce qu'il faudrait évidemment vérifier statistiquement - « un grand nombre de ses thèmes présente une tendance descendante prédominante; pensez par exemple aux principaux motifs de Falstaff, au thème initial de l'Introduction and Allegro, au premier sujet de la Seconde symphonie, et à de nombreux autres ». "Pas étonnant», conclut-il plus loin, "que les Anglais aient le sentiment qu'il y a chez Elgar quelque chose de particulier qui leur est propre, et que les non - britanniques ne peuvent apprécier » (Hall $1953: 6$ ). discours, cette analyse nous rappelle également qu'elle peut être restreinte à une culture particulière. Par un processus spécifique, une langue peut laisser sa marque dans la musique de la même société. Utilisant le seul texte dont on sait qu'il est resté inchangé tout au long de l'histoire de la musique occidentale, Thrasybulos Georgiades (1954) a montré comment chaque époque a traité les paroles canoniques de la Messe, et comment, par comparaison, le style de chaque pays a été influencé par les structures, notamment rythmiques et accentuelles, de la langue correspondante. Mais surtout, il faisait la démonstration que les musiques instrumentales, en s'émancipant progressivement de la musique vocale dominante jusqu'au baroque, ont conservé la trace des langues avec lesquelles elles avaient été en contact pendant au moins dix siècles.

Le mot qui vient spontanément sous la plume d'auteurs qui reconnaissent à la musique une sorte de "narration en creux », est celui de geste (même si cette dénomination n'est qu'une approximation), sans doute parce que, comme disait Combarieu au début du XXème siècle, "la musique nous émeut parce qu'elle nous incite à nous mouvoir ». Aussi, dans son ouvrage récent consacré à la sémantique musicale, Ole Kühl a rebaptisé " geste » ce qui le plus souvent n'est pas autre chose qu'une unité musicale, analogue aux motifs et aux phrases, mais considéré du point de vue de leur dimension expressive (Kühl 2008: chap. VIII). "Le geste mahlérien est celui de l'épopée », écrit encore Adorno (1976: 95), même s'«il reste interdit à la musique épique de décrire le monde qu'elle vise » (ibid., p. 108). Dans cette perspective, il paraît pertinent d'expliquer le succès universel du Boléro de Ravel, non pas seulement par la simplicité de sa structure répétitive, mais par sa capacité à évoquer la montée irrésistible du désir jusqu'à l'éclatement orgasmique des trombones, comme l'a bien vu Maurice Béjart dans deux chorégraphies justement célèbres.

Une comparaison avec l'histoire que j'ai invoquée tout à l'heure pour définir le concept d'intrigue, illustrera encore mieux le problème. Les faits historiques ne constituent pas, en eux-mêmes, un récit. Ils sont pris en charge par un récit qui leur donne sens, ce qui est très différent. Comme l'a très clairement montré Paul Veyne (1971 : chap. 2), je peux bien constater que des événements ont eu lieu - une bataille a éclaté, un traité a été conclu, des frontières ont été modifiées -, mais il n'y a interprétation historique proprement dite que lorsque je suis capable, en construisant une intrigue, d'établir entre les événements une relation de causalité qui les explique, c'est-à-dire en fait, de relier entre eux, par la logique d'un récit, les événements retenus. S'il en est ainsi, c'est parce que les événements sont comme des objets neutres inscrits dans le temps et qui, comme tels, sollicitent l'interprétation qu'élabore une narration. Mais pas plus en musique qu'en histoire, ces objets neutres ne sont constitutifs, per se, d'un récit. La narration construite 
à leur sujet occupe une position métalinguistique par rapport aux données ou aux événements que, pour reprendre la précieuse distinction de Hayden White (1965:2), ils ne narrent pas, ils narrativisent, tout comme, au-delà de la sémantique affective inhérente à toute musique, le langage verbal contribue à sémantiser la musique. L'auditeur, lorsqu'il adopte une conduite d'écoute narrative, ou le musicologue, lorsqu'il étudie la musique d'un point de vue narratologique, ne font pas autre chose.

Kühl définit le principe de la narrativité musicale à la fois comme notre pulsion intérieure à donner une cohérence à une expérience et le fait de donner un sens à une série d'événements sonores (Kühl 2008 : 210-211). C'est ce que fait l'analyste de la musique qui décrit et relie entre eux tous les événements musicaux inscrits dans la durée de son déroulement. Quand nous analysons la musique dans sa dimension immanente, nous faisons bel et bien appel à une intrigue dictée par la méthodologie que nous avons adoptée, celle qui nous fait privilégier tel ou tel aspect de la musique, de la même façon que, dans les termes de Veyne, l'historien choisit parmi l'infinité des événements soumis à l'interprétation historique, ceux qui sont pertinents en fonction de la trame explicative qu'il a élaborée.

21 Mais Kühl, un peu dangereusement, n'hésite pas, tout au long de son livre, à parler de narration musicale et de qualifier le déroulement implicatif et linéaire de la musique de " chemin ", même si, s'approchant de la fin de son livre, il concède que l'expression "Les chemins sont des histoires » est une métaphore (ibid.: 216) et si, par une volte-face rhétorique, il déclare avant de conclure: «Du point de vue d'une position sémiocognitive, il semble nécessaire de mettre au clair que, lorsque je parle de gestes, d'agents et de narrations en musique, je le fais métaphoriquement ${ }^{3}$ » (ibid.: 237) C'est cette position soutenue in extremis qui me parait juste.

$\mathrm{Au}$ terme de cet examen, il est sans doute légitime de paraphraser la célèbre formule de Susanne Langer selon qui la musique est an unconsummated symbol (1951:204). Si récit elle est, la musique est un récit incomplet. Mais on ne peut se contenter de cette caractérisation philosophique.

\section{Les origines développementales de la proto- narrativité musicale}

23 L'explication des associations sémantiques et narratives à la musique a fait un progrès considérable lorsque Daniel Stern, dans son ouvrage Le monde interpersonnel du nourrisson, a mis en évidence, dans ses études sur la relation dyadique entre la mère et le bébé, deux constatations importantes placées par Kühl parmi les fondements de sa conception de la sémantique musicale: l'introduction successive et cumulative des différents domaines du lien interpersonnel dyadique et l'existence de modes de relation amodale dès les premières semaines de la vie (Kühl $2008: 59-63^{4}$ ).

Durant la première enfance, chacun des domaines du lien interpersonnel entre la mère et le bébé sont mis en place les uns après les autres mais de manière cumulative, c'est-à-dire que chaque nouveau domaine s'ajoute aux précédents sans les effacer. De la naissance à deux ou trois mois, se met en place le domaine du lien interpersonnel émergent, de 2 ou 3 mois à 7-9 mois, celui du domaine du lien interpersonnel-noyau, de 7-9 mois à 15 , le domaine du lien interpersonnel intersubjectif, et à partir de 15 mois, le domaine du lien interpersonnel verbal. À chaque stade correspond une phase de formation du sens du soi 
(Stern 1989 : 51). Ce qui est capital pour notre propos, c'est que, à chacun de ces quatre stades, vont correspondre dans les premières années de la vie, des formes symboliques spécifiques qui, parce que ces stades se prolongent durant toute l'existence, se retrouveront présentes dans la vie de l'enfant, de l'adolescent et de l'adulte.

Au bout de huit semaines dans la première phase, le contact direct œil à œil s'installe, en même temps que les premiers gazouillis (ibid. : 57) et diverses vocalisations (ibid. :90). L'observation de la succion a permis de voir comment les bébés s'intéressaient à la voix humaine et la préféraient à d'autres types de sons (ibid. : 60). On repère des cris vocaux intentionnels à moins de trois semaines et, entre deux et six mois, des productions de sons dont beaucoup ne font pas partie de sa langue maternelle (Imberty 2004 : 509) et qui peuvent donc être déjà considérées comme musicales. Mais surtout, ce qui est caractéristique de cette période initiale, c'est que le nourrisson est apte "à transférer l'expérience perceptive d'une modalité sensorielle à une autre » (Stern 1989: 70), comme dit Stern, notamment entre le toucher et la vision. Les expériences semblent prouver que cette amodalité est innée et non acquise. Les bébés sont également capables de répondre de manière identique à des signaux d'intensité lumineuse et sonore, ainsi qu'à des motifs temporels sonores et des motifs temporels visuels, et cela, dès l'âge de trois semaines (ibid. : 71-72) : «Le nourrisson paraît avoir une aptitude générale et innée, que l'on peut appeler perception amodale, qui le conduit à traiter des informations reçues dans une modalité sensorielle donnée, et à les traduire dans une autre modalité sensorielle.» (ibid. : 74) Le bébé se fait des représentations abstraites des formes, des intensités et des figures temporelles. «Il est préstructuré pour réaliser certaines intégrations. » (ibid. : 75) Conséquence essentielle : «Certaines propriétés des personnes et des choses, telles que la forme, le niveau d'intensité, le mouvement, le nombre et le rythme sont appréhendées directement comme des attributs perceptifs globaux et amodaux. » (ibid. : 77) Stern en tire la conclusion que naissent alors des « affects de vitalité » qui sont caractérisés par des termes dynamiques et kinétiques comme « surgir », «s'évanouir », fugace », « explosif », « crescendo ", decrescendo », « éclater ", « s'allonger » (ibid. : 78). Prolongement au-delà de la première enfance: "La danse moderne et la musique sont des exemples par excellence de l'expressivité des affects de vitalité » nés à cette période (ibid. : 81). Kühl en tire la conclusion particulièrement pertinente que cela peut, en partie, expliquer l'absence de différences lexicales entre danse, musique et drame dans certaines cultures (Kühl 2008: 61).

Du point de vue qui nous occupe, il y a donc deux choses mises en place avant deux ou trois mois : du côté poïétique, «c'est le réservoir fondamental dans lequel on peut puiser pour toutes les expériences de création.» (Stern, 1989: 95); du côté esthésique, «c'est aussi la source des appréciations affectives des événements en cours » (ibid.). Il n'est pas étonnant alors, si le nourrisson est déjà en possession d'une Gestalt amodale dans laquelle sont réunis les expressions auditives, visuelles et motrices, et comme la liste des termes réunis par Stein le prouve, que les mots utilisés par les sujets des expériences de perception musicale rapportées par Robert Francès (1958) et Michel Imberty (1979) dans leurs différents ouvrages pour tenter de cerner le contenu de la sémantique musicale, relèvent de ces trois modalités, puisque, ainsi qu'ils le démontrent tous deux, la sémantisation repose sur une analogie entre les schémas de tension et de détente, la perception spatio-temporelle de la musique et les réactions musculaires, posturales et gestuelles. Nous tenons ici la racine des potentialités narratives de la musique. 
Les stades ultérieurs du développement des domaines interpersonnels vont mettre en place d'autres formes symboliques qui contribueront à faire de la musique ce qu'elle sera. Après deux ou trois mois, le bébé adresse des vocalisations à d'autres personnes (Stern 1989 : 100) La musique deviendra ainsi un phénomène social, offert à un public et perçu par d'autres intervenants que les créateurs. Il prend également conscience de l'origine des sources sonores (ibid. : 112), ce que, selon les termes du compositeur canadien Murray Schafer, on peut appeler la schyzophonie inhérente à la pratique et l'écoute de la musique. C'est aussi l'âge où le bébé commence à se mouvoir dans une «structure temporelle de base " et à prendre conscience de l'existence du temps (ibid. : 114), une donnée essentielle de la production comme de la perception de la musique. C'est le moment où le nourrisson saisit qu'il y a un rapport entre deux événements de même structure temporelle (ibid. : 116) : c'est là que réside la naissance de la protonarrativité de la musique. J'y vois la naissance du sens du rythme et des affects qui lui sont reliés puisque cette structure temporelle de base est commune à tous les stimuli (auditifs, visuels, tactiles, proprioceptifs). Les chercheurs ont également constaté une structure de l'intensité commune à la force de la vocalisation et celle des mouvements qui l'accompagnent, ce que le nourrisson ressent dans la poitrine, les muscles du bras et les cordes vocales (ibid. : 118). Ces constatations font dire à Imberty qu'entre deux et cinq mois, le bébé perçoit les unités musicales sur la base de la proximité et de la similarité et qu'entre quatre et six mois, il est sensibilisé à la structure de la phrase musicale (Imberty 2004 : 510). Cette communauté de structure contribue à ce que les réponses sémantiques empruntent leur vocabulaire aux divers modes de perception, auditifs, tactiles et proprioceptifs.

Enfin, au cours des neuf premiers mois, les relations entre la mère et l'enfant sont fondées sur des processus d'imitation réciproque que l'on peut expliquer aujourd'hui neurobiologiquement par l'existence des neurones-miroirs (Rizzolatti et Sinigaglia 2008) comme l'a bien vu Kühl (2008:125). «Si le nourrisson vocalise, la mère vocalise en retour. » (Stern 1989: 182) Le bébé utilise des formes protolinguistiques d'expression qui, notamment avec le recours à des contours intonatifs dotés de sémantisme, seront à la base de ce que les chercheurs appelleront ultérieurement la proto-narrativité. Mechthild et Hanuš Papoušek (1981) ont particulièrement mis en évidence que le développement d'un échange et d'un dialogue social entre la mère et l'enfant durant les neuf premiers mois - ce que Trevarthen (1993) qualifiera de proto-dialogue ou de proto-conversation se fondait sur des procédés vocaux musicaux que des études ultérieures ont décrits avec beaucoup de précision (Malloch 1999-2000). Ce qui est constitutif du "dialogue musical» entre la mère et l'enfant est, selon les résultats des enquêtes, un événement vocalement exprimé, fait du début ou de la fin d'une vocalisation dont l'amplitude est spécifique, d'un moment plus fort ou d'un changement dans le contour des hauteurs de la voix de la mère. La mère utilise certains mots auxquels l'enfant répond par des vocalisations, le tout s'inscrivant dans un espace de temps mesurable qui manifeste une remarquable régularité témoignant d'un processus de communication musicalement réglé. Ces vocalisations ainsi délimitées présentent un contour mélodique et timbral spécifique, et l'observation des contours utilisés respectivement par la mère et le bébé démontre l'influence de l'interaction qui s'établit entre eux. Par exemple, une fois que le bébé a procédé deux fois à une vocalisation, avec une deuxième plus haute que la première, la mère continue et exagère le mouvement ascendant dans sa vocalisation subséquente : c'est bien le contour qui est le paramètre pertinent. Il en va de même quand le bébé 
utilise un contour plus plat: la mère répond par un contour similaire. L'ensemble des contours enregistrés démontre un équilibre dans la forme générale des contours alternativement utilisés par la mère et le bébé. Nous tenons ici la racine développementale du fondement intonatif de la sémantique et de la protonarrativité musicales présenté plus haut.

Mais il y a plus. C'est à une véritable construction que l'on est confronté et, pour ma part, je n'hésiterais pas à parler ici de la création d'une œuvre collective (ici, avec deux auteurs), analogue à celle qui résulte de l'interaction entre les musiciens de jazz. Ce n'est d'ailleurs pas un hasard si une psychologue de la musique, Maya Gratier, elle-même impliquée dans l'étude des relations dyadiques entre la mère et le bébé, y a vu le fondement des relations à l'œuvre dans une exécution de jazz (Gratier 2008). Une étude de Stephen Malloch s'attarde également à l'organisation musicale des relations de timbre dans la vocalisation de la mère et du bébé. Cette fois-ci, les mesures démontrent la recherche, chez l'une et chez l'autre, d'effets de contraste. Tout cela, dit Malloch, contribue à créer, entre la mère et son enfant, "la structure narrative de leur camaraderie (companionship)»(1999-2000: 45), même si une fois encore, il serait préférable de parler ici de proto-narration, d'autant plus que l'auteur définit alors le récit comme ce qui permet à deux personnes «de partager le sens du temps qui passe et de créer et de partager les enveloppes émotionnelles qui évoluent au travers de ce temps partagé » (ibid.).

30 Et c'est bien d'expression musicale qu'il s'agit: tout comme dans la musique que nous connaissons, les imitations font l'objet de modifications et Stern n'a pas hésité à parler d'une forme «thème et variations » dans la mesure où chaque nouvelle vocalisation peut être différente. (Stern 1989: 183) Le bébé reproduit par imitation et transformation les constituants du «baby-talk», du "parler-bébé » de la mère: les procédures de segmentation, la répétition, la simplicité syntaxique, la lenteur du tempo, et la simplification et l'amplification des patterns expressifs des contours mélodiques, recourant à cinq ou six prototypes de courbes intonatives, ce qui fait dire à Imberty, dans la foulée de Stern, que la répétition de ces patterns « engendre une régularité qui permet au sujet d'anticiper le cours du temps " (Imberty 2004 : 511-513). On est sans doute à la source, non seulement du mécanisme répétition-transformation que la technique paradigmatique de Ruwet permet de décrire, mais de la relation implication-réalisation analysée par Meyer. Tous ces procédés contribuent à ce que, dès le sixième mois, commence à se développer chez le bébé un sens de l'autre fondé sur la communication intentionnelle.

31 Tout est donc en place pour le développement de ce qui sera appelé ultérieurement chez divers auteurs, la proto-narrativité musicale et qui permettra l'accordage entre la mère et l'enfant, recourant à l'intensité, le rythme et la forme, paramètres dont il faut se souvenir qu'ils sont transmodaux (Stern 1989: 190, 192, 197-199). «La plupart des comportements humains, écrit Stern, se composent de formes cinétiques - c'est-à-dire de configurations changeants au cours du temps - et les vocalisations sont l'une des formes cinétiques les plus fréquentes dans les accordages. " (ibid. : 199) Et ce sont des réponses à caractère cinétique que l'on trouve souvent dans les verbalisations sur la sémantique musicale obtenues par les expérimentalistes.

Une fois entré dans la dernière phase de la petite enfance, l'être humain va pouvoir commencer à recourir au langage pour caractériser ce qu'il ressent, y compris en écoutant de la musique. 

la mère et le bébé ont conduit Stern, dans un ouvrage ultérieur (La constellation maternelle), à développer la notion d' « enveloppe proto-narrative » (1995: 82-93) qu'il a décelée chez le bébé lorsqu'il organise les premières formes de son rapport au temps, sur la base, par exemple, de l'expérience de l'attente, du désir de nourriture ou de l'interaction avec la mère. Les schémas d'enveloppes proto-narratives qu'il publie, montrent très bien que l'intensité du désir de l'enfant débute avec l'entrée de sa mère dans la chambre, croît jusqu'à ce qu'elle offre le mamelon de son sein au bébé, et que la courbe de cette intensité décroît jusqu'à ce que le bébé soit satisfait du lait qu'il a pu consommer (Stern 1995 : 86). «Lorsque la motivation (désir) est activée en une situation interpersonnelle, elle crée subjectivement une structure quasi narrative» (Stern 1995 : 19). Cette enveloppe, expliquent Jean et Raphaël Molino, qui renvoient à Stern dans Homo Fabulator, «est à la fois la première forme d'organisation du temps et le fondement psychologique de l'intrigue vécue ${ }^{5}$. On comprend alors l'importance du suspens dans le récit : celui-ci est organisé à tous les niveaux, du plus local au plus global, par cette même structure de tension et de détente, que met en évidence la fin de chaque épisode d'un roman-feuilleton » (Molino, Lafhail-Molino 2003: 250). Il est particulièrement remarquable que les termes de "tension» et de "détente» puissent s'appliquer également au phénomène musical. S'inspirant de Labov, Stern nous rappelle que les formes premières de causalité et une "ligne de tension dramatique » font apparaître ce qu'il appelle une intrigue ou une proto-intrigue (Stern 1994) et, comme le précise Imberty, «[elles] font émerger de la trame temporelle du ressenti une ligne de tension dramatique qui l'oriente » (Imberty 2004 : 522). Aussi cette expérience du nouveau-né et de l'enfant peut être également à la base de notre rapport à la musique dans son déroulement linéaire fait d'attentes et de résolutions, mutatis mutandis bien entendu. Dans les deux cas, aussi bien l'enveloppe proto-narrative vécue par le nourrisson et le schéma dans lequel s'inscrit le développement musical sont caractérisés par un contour inscrit dans le temps, avec le temps en abscisse et l'intensité de l'attente et de sa résolution en ordonnée. De plus, il est particulièrement remarquable de noter que, si dans cette présentation des propositions de Stern, je suis allé du concept de proto-narrativité chez le nourrisson à la proto-narrativité musicale, en fait, dans son exposé, c'est la musique qui lui a servi de modèle et à propos de laquelle il parle d'enveloppe proto-narrative «Une des plus grandes surprises de la recherche récente sur les nouveau-nés a été de découvrir que les bébés n'ont besoin ni de mots ni de symboles pour se représenter différentes formes sonores, visuelles ou tactiles, comme on le pensait auparavant» (Stern 1998 : 170). «Nous supposons que [les] enveloppes proto-narratives sont les fondements de la connaissance implicite contenue dans la connaissance humaine relationnelle. Ce qui est intéressant [...] est le fait que les unités subjectives les plus petites (les moments présents), desquelles dépend l'appréciation affective de la musique, sont d'un concept très similaire aux unités subjectives de base sur lesquelles est construite une compréhension des relations interpersonnelles non-verbales. » (ibid. : 182-183)

Plutôt que de voir dans le jeu des configurations musicales de la tension et de la détente une forme de narration, il me semble donc beaucoup plus fructueux de suivre les analyses des psychologues de la musique qui, inspirés par Stern, se sont attardés précisément à montrer comment ils fondent leur interaction proto-narrative sur les unités inscrites dans le temps, les contours, les schémas intonatifs, ceux-là même que, plus tard, la musique utilisera pour imiter l'allure du récit, et qui ont conduit certains des auteurs des 
numéros de Musicae Scientiae, notamment Imberty et Gratier, à parler de "protonarrativité musicale». C'est pourquoi, en me fondant sur les observations de la psychologie développementale, je propose fermement de renoncer à parler de récit musical et d'adopter l'expression de proto-récit.

\section{Le discours de la musicologie narratologique comme récit de fiction}

35 J'ai, par le passé, affiché une certaine distance vis-à-vis des travaux de ce que l'on peut appeler la musicologie narratologique, mais sans jamais rentrer dans le détail de mes critiques. Dans la foulée de la section précédente, je résumerai en ces termes la position que je vais tenter d'étayer maintenant: l'attitude de cette orientation de la musicologie contemporaine a l'inconvénient de confondre le fonctionnement sémiologique des configurations musicales avec le contenu des conduites esthésiques qui les narrativisent, nécessairement métalinguistiques. Céder alors à la tentation de parler de "récit musical ", c'est glisser d'une métaphore - en elle-même suggestive si on n'oublie pas que c'est une métaphore comme le reconnaît Kühl - à une illusion ontologique selon laquelle, parce que la musique suggère le récit, elle serait elle-même "un art narratif », comme Tarasti la qualifie (2007). C'est cette nuance qu'on pourrait croire ténue, en réalité essentielle, qui fait toute la différence entre le récit littéraire et le proto-récit musical.

Suite aux échanges du colloque de Lausanne tenu le 28 octobre 2011 et dont la présente livraison est le prolongement, je crois nécessaire de préciser ma position.

Je n'ai évidemment pas de problème de principe avec l'intérêt des musicologues narratologiques pour les musiques explicitement narratives du point de vue poïétique, dont les prototypes sont Les quatre saisons de Vivaldi ou les poèmes symphoniques, ou celles qui ont été composées, non pas pour, fidèlement, s'inspirer musicalement d'un texte littéraire, mais pour suivre un fil narratif plus ou moins précis, celui que suggère les titres des cinq mouvements de la Symphonie pastorale de Beethoven, par exemple, ou celui que l'on peut reconstituer dans les symphonies de Mahler en s'appuyant sur les documents ou les propos laissés par le compositeur ${ }^{6}$.

Ce qui peut faire problème, dans l'approche des musicologues narratologiques des musiques explicitement narratives, ce sont les méthodes suivies pour reconstituer l'intention narrative qui les sous-tendent. Je ne les aborderai pas ici. Si cette intention peut être définie poïétiquement, dans le meilleur des cas avec les techniques éprouvées de l'investigation historique et philologique, en revanche, ce que, du point de vue esthésique, les spectateurs et auditeurs en perçoivent, selon qu'ils ont accès ou non au programme, au titre ou aux paratextes linguistiques inscrits dans la partition, ne pourra être établi de manière rigoureuse qu'en recourant aux méthodes expérimentales. Pour savoir quelle histoire les auditeurs entendent dans une œuvre instrumentale dont l'intention narrative est attestée, il convient tout simplement de le leur demander en omettant de leur faire part du titre et du programme de l'œuvre ${ }^{7}$.

Je tiens à souligner, en deuxième lieu, que les travaux de la musicologie narratologique ont le grand mérite de remettre à l'ordre du jour les recherches entamées par Leonard Ratner (1980) et poursuivies par Robert Hatten (1994) et Raymond Monelle (2000) qui démontrent l'existence d'un niveau d'organisation de l'œuvre constitué d'unités porteuses d'affects ou de figures topiques, distincts des structures élémentaires 
traditionnelles, comme les degrés de l'échelle utilisée, les structures mélodicorythmiques et harmoniques, la phraséologie ou les grandes sections formelles. Ces investigations sont parallèles à celles de Boris Asafiev et de József Ujfalussy, présentées et commentées par Grabócz (2009). Je reproduis ici la définition du «topos » proposée par Monelle et qui me semble particulièrement éclairante: « Nous assimilons les topiques à des fragments de mélodie ou de rythme, des formes conventionnelles ou encore des aspects du timbre ou de l'harmonie qui désignent des éléments de la vie sociale ou culturelle, et par conséquent des thèmes comme la virilité, la campagne, l'innocence, la plainte, etc. » (Monelle 2007 : 178), ou, comme il le dit ailleurs : «Une figure devient un topos quand son évocation devient conventionnelle.» (Monelle 2001 : 105). Ce n'est pas le lieu de rentrer ici dans le détail du contexte épistémologique dans lequel ces auteurs se situent, oscillant entre l'herméneutique la plus classique et les préoccupations des divers paradigmes sémiologiques, ni de discuter les méthodes qu'ils utilisent pour définir les unités musicales considérées comme topoi et pour déterminer les significations qui leur sont associées. Qu'il me suffise, pour l'heure, de souligner que le grand mérite du concept de topos est de signaler qu'il existe des classes de configurations symboliques (au sens de Piaget et Cassirer) à la disposition des compositeurs durant une période de temps assez longue, par exemple le topos du ranz des vaches (ou de l'air de berger) dont parle Rousseau dans son Dictionnaire de musique et que l'on retrouve aussi bien chez Grétry, Beethoven, Rossini, Berlioz, Liszt, Schumann et Wagner. La notion de topos me semble donc être un apport remarquable à la dimension poïétique de la sémantique musicale, complétant les travaux des psychologues expérimentalistes (Francès, Imberty) qui, eux, se situent expressément du côté de l'esthésique (et qui sont malencontreusement et totalement ignorés des musicologues narratologiques $\left.{ }^{8} . ..\right)$.

Il faudrait entreprendre une étude minutieuse pour déterminer la part de "narrativité ", au sens précis du terme, que les musicologues narratologiques pensent discerner dans la musique et en quoi elle consiste musicalement. On les sent plus d'une fois prudents et l'hésitation d'un Kofi Agawu est révélatrice à cet égard. Dans son ouvrage Playing with Signs, il fournit une liste éloquente de 27 topoi pour la musique classique : « alla breve, alla zoppa, amoroso, aria, bourrée, brillant style, cadenza, sensibility, fanfare, fantasy, French overture, gavotte, hunt style, learned style, Mannheim rocket, march, minuet, musette, ombra, opera buffa, pastoral, recitative, sarabande, sigh motif, singing style, Sturm und Drang et Turkish music. » (Agawu 1991 : 30), mais il précise d'emblée : « On n’a pas encore démontré, sinon de manière extrêmement triviale, que la musique ait la capacité de raconter » (1991 : 36). Pourtant, ultérieurement, dans son livre Music as Discourse (Agawu 2009), à propos de Beethoven, Brahms et Mahler, il cède lui aussi à la tentation narratologique comme l'indique le titre de son livre', et il n'est pas le seul. (Je pense ici aux contributions de Lawrence Kramer, Anthony Newcomb et Leo Treitler.).

Ce sont aux travaux d'esprit narratologique appliqués aux musiques instrumentales «pures » que je voudrais m'attarder. À propos de mes réticences à parler de "récit » musical, Grabócz écrit à mon sujet: «Ce qui relie toutes ces applications de tel ou tel modèle n'est pas la volonté de voir une "histoire racontée en musique", comme le présuppose Nattiez, mais de trouver les règles, les stratégies d'organisation des signifiés " (2009 : 27). Est-ce que je ne saurais pas lire ?! Eero Tarasti affirme dans sa Sémiotique musicale: "Par narrativité en musique, [nous entendons] la narrativité "structurelle" selon laquelle toute pièce musicale qui se déploie dans le temps, et qui transforme quelque chose en autre chose, doit être considérée comme narrative ${ }^{10} »$ (Tarasti 1996 : 
406). Dans le recueil Sens et signification en musique édité par Grabócz, il récidive et intitule sa contribution « La musique comme art narratif ». "La musique constitue un art narratif à part entière ${ }^{11}$ ", déclare-t-il dès le premier paragraphe. Et Grabócz elle-même, à propos de l'Ouverture Leonore No. 3 de Beethoven, ne parle-telle pas de sa «syntaxe discursive » (Grabócz 2009: 191) ? Alors, la musique, elle est porteuse d'un récit, oui ou non?! Ces deux auteurs m'opposeront sûrement les propos par lesquels ils semblent vouloir corriger le tir ou nuancer leur position. Tarasti : «L'approche sémiotique que je souhaite développer dans cet article ne prétend pas démontrer que la musique est capable d'énoncer des récits spécifiques, mais expose plutôt en quoi des structures de la musique peuvent être associées ${ }^{12}$ à des récits. » (Tarasti 2007 : 209) Cela est fort différent, car, dès lors, ce n'est plus la musique qui, en elle-même et par elle-même, serait « un art narratif ». Grabócz: «Nous appelons "narrativité musicale" la concaténation des topiques" (Grabócz 2009: 28). Mais qui donne un sens narratif à cette organisation syntagmatique? Certainement pas cette seule succession, sinon une œuvre musicale serait capable, par elle-même, de nous dire: "Je suis l'Ange gardien, la Muse et la Madone »! Une concaténation de moments ne parle pas toute seule. En fait, dans un cas comme dans l'autre, la musique suscite l'énoncé d'un récit de la part des auditeurs ou... des musicologues qui, pour ce faire, ont recours aux catégories métalinguistiques de la narratologie générale. Si les narratologues de la musique semblent jouer double jeu, c'est parce qu'ils n'ont pas reconnu la nature protonarrative (et non narrative) de la musique.

Les travaux de Grabócz et de Tarasti présentent fondamentalement trois grandes difficultés. D'une part, ces chercheurs semblent considérer que, s'il est possible d'appliquer (en admettant que ce soit le cas, je vais y revenir) les modèles de la narratologie littéraire à la musique instrumentale "pure», ce serait la preuve que la musique est bien un «art narratif ». C'est un peu comme si, parce qu'il a été possible, à partir des années 1960, d'appliquer à la musique les modèles de la phonologie, de l'analyse paradigmatique et de la grammaire générative, on avait déduit de cette expansion méthodologiquement fructueuse, que la musique était un langage, au sens du langage verbal.

En deuxième lieu, il est assez stupéfiant que des chercheurs qui se réclament de la sémiotique, en aient ignoré un des principes fondateurs : toute forme symbolique ou tout système de signes (le langage verbal, la musique, le mythe, le cinéma) a des propriétés sémiologiques spécifiques, et on ne saurait confondre l'objet de l'investigation sémiologique et le métalangage qui tente d'en rendre compte. Comme disait Hjelmslev, le langage humain est le seul capable de «parler » tous les systèmes de signes non verbaux.

En troisième lieu, les modèles de la narratologie générale, tout particulièrement ceux de Tzvetan Todorov et d'Algirdas Greimas, sont-ils applicables à la musique? Encore faudrait-il qu'ils soient restés adéquats pour analyser le récit littéraire. Je voudrais évoquer ici le témoignage de François Rastier, co-auteur avec Greimas de l'article historique « Les jeux des contraintes sémiotiques » (Greimas \& Rastier, 1970), qui, lors du $1^{\text {er }}$ congrès de l'Association internationale de sémiotique à Milan, en janvier 1974, prononça un mea culpa remarqué. Prenant sans équivoque ses distances avec le modèle du «carré sémiotique» à l'élaboration duquel il avait participé, il eut l'honnêteté scrupuleuse de rapporter, pour l'endosser, l'irritation agacée des spécialistes de l'Ancien et du Nouveau Testament devant les approches structurales proposées dans le $\mathrm{N}^{\circ} 22$ de la revue Langages, "Sémiotique narrative : récits bibliques ». Comment, en effet, prétendre réduire à des «structures ", des textes dont la philologie a tant de peine à établir la lettre 
et dont l'interprétation doit se fonder - c'est d'ailleurs le propre de l'herméneutique religieuse que d'en creuser le buissonnement-sur la prolifération infinie des interprétants au sens de Peirce? Et que dire de la retractatio, non moins courageuse, de Tzvetan Todorov lui-même, qui, dans Devoirs et délices, s'est livré à une auto-critique en règle des approches structurales de la littérature qu'il avait été l'un des premiers à proposer (Todorov 2002: 105-138 ${ }^{13}$ ) ? Il est encore temps de méditer le témoignage de Roland Barthes qui, très tôt, a dénoncé « la mode structuraliste » et appelé de ses vœux « une critique antistructurale » (Barthes $1975: 149$ et 151).

Tel n'est sans doute pas le cas de Grabócz - qui persiste à projeter sur les œuvres instrumentales les contraintes du " carré sémiotique ", même avec la caution imprudente de Charles Rosen - et Tarasti, même si ce dernier, avec le tournant pris par son Existential Semiotics (2000) finira bien par se heurter à l'incompatibilité entre sa fidélité au modèle de Greimas et «le pari d'une efficacité herméneutique neuve », comme la qualifie Daniel Charles dans l'article qu'il consacre à ce livre, soulignant, avec la perspicacité philosophique qu'on lui connaît, qu'elle se fonde sur la sémiosis infinie de Peirce (Charles 2007 : 137-142), dont le concept d'interprétant me semble incapable de fonder l'approche greimassienne et qui même fournit une base sémiologique solide pour en démontrer l'inadéquation.

47 Alors, quel statut épistémologique donner aux travaux de la musicologie narratologique lorsqu'ils traitent de "narrativité musicale» dans les musiques instrumentales? Delalande, je l'ai rappelé en commençant, me semble avoir établi sur une base expérimentale et de manière définitive, que la "conduite de récit» était une des conduites possibles de perception lors de l'audition d'une œuvre instrumentale. Le musicologue d'obédience narratologique ne fonctionne pas autrement, lorsque, continuateur du premier Todorov ou de Greimas, et confronté à la succession d'événements porteurs d'émotion, d'expression et de significations - en particulier les topoi -, il narrativise leur succession syntagmatique en ayant recours à des catégories et des méthodes désormais obsolètes pour rendre compte du fonctionnement du récit littéraire ${ }^{14}$.

La narratologie musicale consiste alors à projeter sur la musique, considérée a priori «comme art narratif » alors qu'elle n'est qu'un proto-récit, un récit «associé » à la musique, comme dit Tarasti, mais ce récit est celui du musicologue qui démontre une fois encore la capacité des êtres humains à inventer des fictions témoignant d'un degré plus ou moins grand de fabulation ${ }^{15}$.

\section{BIBLIOGRAPHIE}

ADORNO, Theodor W. (1960), Mahler, eine musikalische Physiognomik, Francfort, Suhrkamp; trad. J.L. Leleu et T. Leydenbach, Mahler, une physionomie musicale, Paris, Minuit, 1976.

AGAWU, V. Kofi (1991), Playing with Signs. A Semiotic Interpretation of Classic Music, Princeton, Princeton University Prss. 
AGAWU, Kofi (2009), Music as Discourse. Semiotic Adventures in Romantic Music, Oxford - New York, Oxford University Press.

BARTHES, Roland (1975), Roland Barthes par Roland Barthes, Paris, Seuil.

CARONE, Angela (dir.) (2006 ), La narratololgia musicale. Applicazioni e prospettive, Turin, Edizioni Trauben.

CHARLES, Daniel (2007), « De Heidegger à Tarasti : herméneutique musicale et sémiotique de l'existence », in Grabócz 2007 : 133-154.

CONE, Edward (1974 ), The Composer's Voice, Berkeley, University of California Press.

CORDUBAN, Mihaela (2011), Le premier livre du Clavier bien tempéré de Jean-Sébastien Bach. Rhétorique et sémiologie, Sampzon, Delatour France.

DAHLHAUS, Carl (1997), L'idée de la musique absolue [1978], trad. Martin Kaltenecker, Genève, Contrechamps.

DELALANDE, Francois (1989), « La terrasse des audiences du clair de lune : essai d'analyse esthésique. La prise en comptedes écoutes-types comme points de vue d'analyse ", Analyse musicale, $\mathrm{N}^{\circ} 16$, pp. 75-84.

DELATTRE, Pierre (1966), « Les dix intonations de base du français », French Review, vol. XL, N 1 , pp. 1-14.

DELATTRE, Pierre (1967), « La nuance de sens par l'intonation », French Review, vol. XLI, N 3, pp. 326-339.

DELATTRE, Pierre (1969), «L'intonation par les oppositions », Le français moderne, décembre 1969, pp. 6-13.

FRANCÈS, Robert (1958), La perception de la musique, Paris, Vrin.

GEORGIADES, Thrasybulos (1954), Musik und Sprache; das Werden der abendländischen Musik dargestellt an der Vertonung der Messe, Berlin, Göttingen, Heidelberg, Springer Verlag.

GRABÓCZ, Márta (dir.) (2007), Sens et signification en musique, préface de Daniel Charles, Paris, Hermann.

GRABÓCZ, Márta (2009), Musique, narrativité, signification, préface de Charles Rosen, Paris, L'Harmattan.

GRATIER, Maya (2008), « Grounding in Musical Interaction: Evidence from Jazz Performances », Musicae Scientiae, Special issue, p. 71-110.

GREIMAS, Algirdas Julien, et François RASTIER, 1970, « Les jeux des contraintes sémiotiques » [1968], in A. Greimas, Du sens. Essais sémiotiques, Paris, Seuil, pp. 135-155.

GUIRARD, Laurent (dir.) (2010), Cinquante ans de psychologie de la musique. L'école de Robert Francès, Montauban, Alexitère éditions.

HALL, Robert A. (1953), « Elgar and Intonation of British English », Gramophon, vol. XXXI, No 6, 1953, pp. 6-7.

HATTEN, Robert S. (1994), Musical Meaning in Beethoven. Markedness, Correlation, and Interpretation, preface de David Lidov, Bloomington et Indianapolis, Indiana University Press.

IMBERTY, Michel (1979), Entendre la musique. Sémantique psychologique de la musique [tome I], Paris, Dunod. 
IMBERTY, Michel (1985), «La Cathédrale engloutie de Claude Debussy : de la perception au sens », Revue de musique des universités canadiennes, $\mathrm{N}^{\circ}$ 6, pp. 90-160.

IMBERTY, Michel (2004), « Le bébé et le musical », in Musiques. Une Encyclopédie pour le XXIe siècle, Jean-Jacques Nattiez (dir.), Arles-Paris, Actes Sud - Cité de la musique, vol. II, « Les savoirs musicaux », pp. 506-526.

IMBERTY, Michel, GRATIER, Maya (dir.) (2008): « Narrative in Music and Interaction », Musicae Scientiae, Special issue.

IMBERTY, Michel, GRATIER, Maya (2008), « Narrative in Music and Interaction. Editorial », Musicae Scientiae, Special issue, p. 3-13.

JUSLIN, Patrick N., et John A. SLOBODA (dir.), (2010), Handbook of Music and Emnotion. Theory, Resedarch, Applications, Oxford - New York, Oxford University Press.

KÜHL, Ole (2008) [2007], Musical Semantics, Berne, Peter Lang.

LANGER, Susanne K. (1951) [1942], Philosophy in a New Key, New York, Mentor Book.

MALLOCH, Stephen N. (1999-2000), « Mothers and Infants and Communicative Musicality », Musicae Scientiae, Special issue, Numéro spécial, « Rhythm, Musical Narrative, and Origins of Human Communication », p. 29-57.

MATTHESON, Johann, 1954 [1739], Der vollkommene Capellmesiter, Chistian Gerold, Hamburg; facsimilé, Kassel, 1954.

MEYER, Leonard B.(1956), Emotion and Meaning in Music, Chicago, Chicago University Press; trad. Catherine Delaruelle, Emotion et signification en musique, préface de Jean-Jacques Nattiez, Arles, Actes Sud, 2010.

MEYER, Leonard B. (1973), Explaining Music. Essays and Explorations, Berkeley - Los Angeles Londres, University of California Press.

MOLINO, Jean (2009), Le singe musicien. Sémiologie et anthropologie de la musique, préface de JeanJacques Nattiez, Arles, Actes Sud / INA.

MOLINO, Jean, Raphaël LAFHAIL-MOLINO (2003), Homo Fabulator. Théorie et analyse du récit, préface de Gilles-Gaston Granger, Montréal - Arles, Leméac - Actes Sud.

MOLINO, Jean, Joelle TAMINE(1987), Introduction à l'analyse de la poésie, vol. I, Paris, Presses Universitaires de France.

MONELLE, Raymond (2000), The Sense of Music. Semiotic Essays, préface de Robert Hatten, Princeton et Oxford, Princeton University Press.

MONELLE, Raymond (2001), « Horn and trumpet as topical signifiers », Historic Brass Society Journal, vol. XIII, No. 1, p. 102-117.

MONELLE, Raymond (2007), "Sur quelques aspects de la théorie des topiques musicaux », in Grabócz 2007, pp. 177-193.

NATTIEZ, Jean-Jacques (2008), Lévi-Strauss musicien. Essai sur la tentation homologique, Arles Actes Sud.

NATTIEZ, Jean-Jacques (2010), La musique, les images et les mots. Du bon et du moins bon usage des métaphores dans l'esthétique comparée, Montréal, FIDES.

NATTIEZ, Jean-Jacques, Sylvia NANNYONGA-TAMUSUZA (2005), « Rythme, danse et sexualité : une danse ougandaise d'initiation au mariage », in Musiques. Une Encyclopédie pour le XXIe siècle, 
Jean-Jacques Nattiez (dir.), Arles-Paris, Actes Sud - Cité de la musique, vol. III, « Musique et cultures », pp. 1108-1129.

NETTL, Bruno, 1958: « Some Linguistic Approaches to Musical Analysis », Journal of the International Folk Music Council, vol. X, p. 37-41; trad. N. Scotto di Carlo, D. Sales, « De quelques méthodes linguistiques appliquées à l'analyse musicale », Musique en jeu, $\mathrm{N}^{\circ}$ 5, 1971, pp. 61-66. PAPOUŠEK, Mechthild et Hanuš, 1981: « Musical Elements in the Infant's Vocalizations: their Significance for Communication, Cognition and Creativity », in Advances in Infancy Research, L.P. Lipsitt (dir.), Norwood, Ablex, vol. I, pp. 163-224.

RATNER, Leonard G. (1980), Classic Music. Expression, Form, and Style, New York, Schirmer.

RIZZOLATTI, Giacomo, Corrado SINIGAGLIA (2008), Les neurones miroirs, Paris, Odile Jacob.

STEFANI, Gino, Stefania GUERRA LISI (2009), Les styles prénatals dans les arts et dans la vie, Paris, L'Harmattan.

STERN, Daniel (1985), The Interpersonal World of the Infant. A View from Psychoanalysis and Developmental Psychology, New York, Basic Books; trad. Alain Lazartigues et Dominique Pérard, Le monde interpersonnel du nourrisson. Une perspective psychanalytique et développementale, Paris, Presses universitaires de France, 1989.

STERN, Daniel (1994), « Une manière de construire un nourrisson cliniquement pertinent », Infant Mental Health Journal, vol. XV, $\mathrm{N}^{\circ}$ 1, pp. 9-25.

STERN, Daniel (1995), The Motherhood Constellation. A Unified View of Parent-Infant Psychotherapy, New York, Basic Books; trad. D. Cupa, La constellation maternelle, Paris, Calmann-Lévy, 1997.

STERN, Daniel (1998), « Aspects temporels de l'expérience quotidienne d'un nouveau-né : quelques réflexions concernant la musique ", in Le temps et la forme. Pour une épistémologie de la connaissance musicale, Étienne Darbellay (dir.), Genève, Droz, pp. 167-189.

TARASTI, Eero (1996), Sémiotique musicale [1994], Limoges, Presses universitaires de Limoges.

TARASTI, Eero (2000), Existential Semiotics, Bloomington, Indiana Univesity Press.

TODOROV, Tzvetan (2002), Devoirs et délices. Une vie de passeur. Entretiens avec Catherine Portevin, Paris, Seuil.

TREVARTHEN, Colwyn (1993), « The Function of Emotions in Early Infant Communication and Development », in J. Nadel et L. Camaioni (dir.), News Perspectives in Early Communicative Development, Londres, Routledge, pp. 48-81.

TREVARTHEN, Colwyn (dir.), 1999-2000: « Rhythm, Musical Narrative, and Origins of Human Communication », Musicae Scientiae, Special issue, Numéro spécial, 1999-2000.

VEYNE, Paul (1971), Comment on écrit l'histoire, Paris, Seuil.

WHITE, Hayden (1965), Foundations of Historical Knowledge, New York, Harper and Row.

\section{ANNEXES}

\section{Biographie}

Jean-Jacques Nattiez est professeur émérite de musicologie à la Faculté de musique de l'Université de Montréal. Considéré comme pionnier de la sémiologie musicale, il a publié 
Fondements d'une sémiologie de la musique, Musicologie générale et sémiologie, De la sémiologie à la musique, Le combat de Chronos et d'Orphée. Il a appliqué ses concepts sémiologiques à divers sujets : la pensée musicale de Pierre Boulez (préfaces et articles); la musique des Inuit (Canada), des Aïnou (Japon), des Baganda (Ouganda) et des Indiens Nahuas (Mexique) (disques et nombreuses études) ; les relations entre musique et littérature ( Proust musicien). Il est également l'auteur d'un roman (Opera) et d'une autobiographie intellectuelle (La musique, la recherche et la vie). L'œuvre et la pensée de Wagner sont au centre de son travail. Outre des articles, il est l'auteur de Tétralogies (Wagner, Boulez, Chéreau), essai sur l'infidélité; Wagner androgyne ; Les esquisses de Wagner pour Siegfried's Tod. Il a été le directeur général d'une Encyclopédie de la musique en cinq volumes publiée entre 2001 et 2005, en italien, chez Einaudi et de 2003 à 2007, en français, chez Actes Sud, sous le titre général : Musiques. Une Encyclopédie pour le XXIe siècle. Il prépare actuellement des essais sur Wagner antisémite, le solo de cor anglais de Tristan et Isolde, un Traité de musicologie générale et la publication de conférences données en 2006 au Collège de France sous le titre « Unité ou éclatement de la musicologie?».

\section{NOTES}

1. Autre exemple, un slogan significatif de la chaîne musicale de Radio-Canada : "La musique, au-delà des mots, nous raconte une histoire. » (Septembre 2011)

2. Pour des commodes recueils de textes illustrant cette tendance, voir Carone 2006 et Grabócz 2007. Pour un bilan, voir Grabócz, «Bref aperçu sur l'utilisation des concepts de narrativité et de signification en musique », in Grabócz 2009 : 21-57.

3. C'est moi qui souligne.

4. Marta Grabócz a eu l'obligeance de me signaler l'existence d'un livre de Gino Stefani et Stefania Guerra Lisi (2009) consacré aux « styles prénatals dans les arts et dans la vie ». Le délai serré de publication du présent article ne m'a pas permis de trouver le temps de me le procurer, mais j'en tiendrai compte, le cas échéant, dans mon Traité de musicologie générale en préparation 5. Je souligne cette expression qui justifie que l'on puisse parler de proto-narrativité entre la mère et le bébé.

6. Voir Dahlhaus 1997 : 123-125.

7. A titre d'exemples, on pourra lire l'enquête d'Imberty sur la perception de la narration à la base de La cathédrale engloutie de Debussy (Imberty 1985 : 153-158) et la mienne auprès de jeunes auditeurs de L'apprenti sorcier de Dukas (Nattiez 2010 : 113-119).

8. Mais il n'est pas trop tard pour bien faire. Un récent ouvrage, Cinquante ans de psychologie de la musique. L'école de Robert Francès (Guirard 2010) vient de souligner, dans une perspective élégamment critique, l'importance et les prolongements de La perception de la musique de Francès (1958). Un article d'Imberty y fait le lien entre les recherches de sémantique musicale et les travaux de la psychologie développementale dont j'ai parlé plus haut. Et il serait peut-être urgent que la musicologie narratologique se plonge dans les 975 pages du Handboo of Music and Emotion (Juslin et Sloboda 2010).

9. Voir en particulier Agawu 2009 : 102-106, 195-196, 239, 247, 259, 247, 255, 271-273, 278-279.

10. C'est moi qui souligne.

11. C'est moi qui souligne.

12. Ces mots sont soulignés par l'auteur.

13. Faut-il rappeler que, dans ce monument d'érudition et de perspicacité épistémologique qu'est le Homo Fabulator de Molino et Lafhail-Molino (2003), on trouvera un précieux antidote aux 
dérapages des approches structuralistes de la littérature, à condition évidemment de ne pas décider, tout simplement, de l'ignorer.

14. Il conviendrait évidemment d'entreprendre une critique élaborée du « carré sémiotique » de Greimas, référence méthodologique de Grabócz et Tarasti,. Pour comprendre dans quelle perspective épistémologique et critique je me situe de manière générale par rapport au structuralisme des formes symboliques littéraires et mythologiques, on pourra consulter mon Lévi-Strauss musicien (Nattiez 2008 : chap. 4 et 5).

15. En comparaison de la conférence que j'ai présentée à Lausanne le 29 octobre 2011, le présent texte a bénéficié d'échanges stimulants et provocants avec Márta Grabócz, Françoise Revaz et Raphaël Baroni au cours du colloque, et de communications personnelles particulièrement fructueuses de Jean Molino.

\section{RÉSUMÉS}

Après avoir rappelé les grandes caractéristiques du récit littéraire et montré les analogies avec la musique, l'auteur insiste sur la nécessité de ne pas la considérer a priori comme un récit. Il analyse l'intonation des contours musicaux comme une forme de proto-narration qui est ensuite expliquée par les découvertes de la psychologie développementale, notamment dans les travaux de Daniel Stern. Il est alors fermement proposé de parler de proto-récit musical et, sur cette base, l'auteur développe une critique de la musicologie narratologique.

After describing the main features of the literary narrative and demonstrating its analogy with music, the author underlines the necessity not to conider a priori a musical production as a narrative. He analyzes the musical intonation contours as a form of proto-narration which he later explains from the standpoint of Daniel Stern's developmental psychology. It is then emphasized that music should be considered as a proto-narrative and the authors proposes a criticism of the so-called narratological musicology.

\section{INDEX}

Mots-clés : musicologie narratologique, psychologie développementale, sémiologie, contours, intonation, Marta Grabócz, Daniel Stern, Eero Tarasti

\section{AUTEUR}

JEAN-JACQUES NATTIEZ

Université de Montréal 